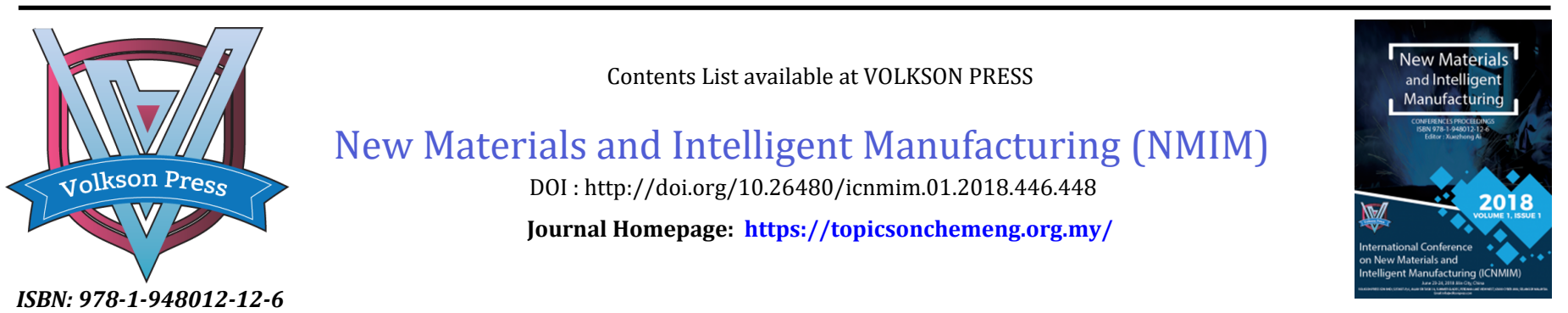

\title{
STUDY ON EXTRACTION OF CORN STRAW AND CORN CELLULOSE AND THE DEGRADATION PERFORMANCE OF REGENERATED FILM
}

\author{
Wang ben, Pan gaofeng, Fu qiang*, Li yi, Xue daliang \\ Jilin Institute of Chemical Technology, Institute of Petrochemical Technology, Chengde Street No. 45, Jilin, China \\ *Corresponding Author E-mail: xia03368860@163.com
}

This is an open access article distributed under the Creative Commons Attribution License, which permits unrestricted use, distribution, and reproduction in any medium, provided the original work is properly cited

\section{ARTICLE DETAILS}

\section{Article History:}

Received 26 June 2018 Accepted 2 July 2018

Available online 1 August 2018

\section{ABSTRACT}

In this paper, corn straw and corn cob are sifted into outer layer and inner layer, respectively. Then, the cellulose was separated by acid and alkali method, dissolved in ionic liquid AmimCl, cellulose film was regenerated in water from the solution of cellulose/AmimCl. At last, the cellulose content, degree of polymerization (DP) and the degradation were studied. This study provides a chance to prepare Functional cellulose Regenerated film using relatively cheap and large amount of corn byproduct as cellulose resources. The result showed that the content of cellulose in the outer layer of corn straw is higher than the inner layer. The cellulose content in the outer layer of corn cob is higher than that in the inner layer of corn cob; The Dp of corn straw cellulose is the highest among corn straw cellulose, corn cob cellulose and corn leaf; Corn straw regenerated film can be degraded in the soil and completely degraded in just 15 days.

\section{KEYWORDS}

corn straw, corn cob, cellulose, degradation.

\section{INTRODUCTION}

With the depletion of non-renewable resources such as petroleum and coal, it has become a research hotspot to make high added value chemical products from renewable biomass, especially waste biomass. Corn is one of the most widely distributed crops in the world. China is a large corn planting country, mainly concentrated in the three northeastern provinces and Inner Mongolia. According to 2011 data from the National Bureau of Statistics, the planting area and yield of corn in Jilin Province were $3.1 \times$ $10^{6}$ hectares and $2.3 \times 10^{7}$ tons, respectively, accounting for $10 \%$ and 12 $\%$ of the total corn in the country. However, most of the agricultural waste brought about by the corn industry, corn stalks and corn cores, is used for burning for heat, which not only wastes energy but also pollutes the environment. Corn straw and corn cob contain a lot of cellulose and make full use of renewable cellulose to produce high-value-added cellulose regeneration and derivative products. However due to the stiff molecule and close chain packing via numerous inter- and intra-molecular hydrogen bonds in cellulose, it is difficult to dissolve cellulose without chemical modification or derivatization. Up to now, Researchers have mainly used the viscose process which had lasted for 100 years and NMMO process which ranks among the new environmentally friendly processes to make regenerated cellulose. While, despite the industrialization of NMMO solvent system, it has some disadvantages associated with its use, such as the requirement for high temperature to dissolve, activation needed before dissolution, the degradation of cellulose, the side reaction of the solvent itself without an antioxidant, and its high cost as well. Therefore, the search for excellent and environment friendly solvents for cellulose continues.

Recently, Room temperature Ionic Liquids have been used to dissolve native cellulose. Based on a study, ILs is advantageous over other solvents due to the following properties: (1) it is a non-derivatizing solvent; (2) it is an environmentally green solvents for a broad range of chemical and industrial process; (3) it can be recycled easily in many cases [1]. Already, 1-butyl-3-methylimidazolium chloride (BmimCl) and 1-allyl-3methylimidazolium chloride $(\mathrm{AmimCl})$ have been reported to be suitable solvents for cellulose.
In this paper, corn straw and corn cob were divided into different parts. Cellulose was extracted separately, and the preparation and degradation properties of regenerated film were studied.

\section{EXPERIMENTAL MATERIALS AND METHODS}

\subsection{Reagents and Instruments}

Corn straw and corn cob was obtained from Jilin city, jilin province, China. It was washed, dried in sunlight, grinding and then dried in the oven. Nmethyl imidazole, purchased from Kaile Chemical Plant in Linhai City, Zhejiang Province, with pure premised; allyl chloride, purchased from Wuhan Xinbao Chemical Co., Ltd., with pure premise; $\mathrm{NaOH}, \mathrm{HNO}$ and other chemical reagents are pure analysis.

The FT-IR spectra of the samples were recorded with a Fourier transform FT-IR spectrometer (Perkin-Elmer GX II, United States of America). The test specimens were prepared by the $\mathrm{KBr}$-disk method. Thirty-two scans were taken of each sample recorded from 4000 to $400 \mathrm{~cm}^{-1}$ at a resolution of $2 \mathrm{~cm}^{-1}$ in the transmission mode.

\subsection{Process for extraction of cellulose}

Corn straw and corn cob are sifted into outer layer and inner layer, respectively. According to research, the extraction method of cellulose is described in the references and the process steps are shown in Figure 1 [2].

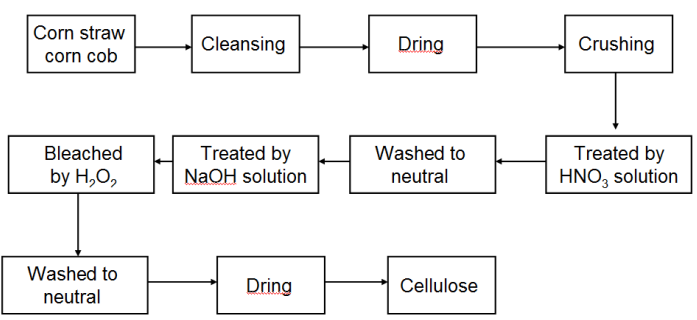

Figure 1: Process for extraction of cellulose 


\subsection{Synthesis of AmimCl}

AmimCl ionic liquids were synthesized from allyl chloride and N-methyl imidazole. The synthesis method is described in the references and the synthesis steps are shown in Figure 2.

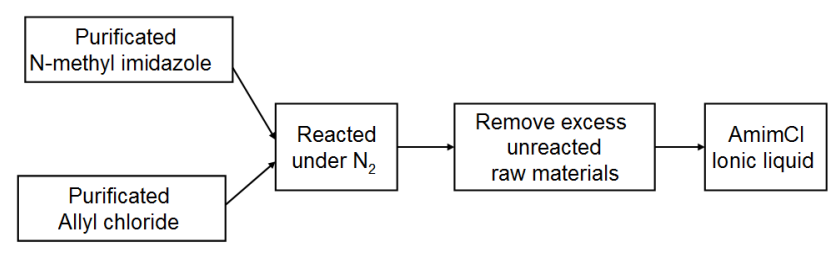

Figure 2: Process for Synthesis of AmimCl

\subsection{Preparation of Cellulose Regenerated Film}

$0.80 \mathrm{~g}$ cellulose and $19.20 \mathrm{~g}$ Ionic liquids ( $4 \%$ mass concentration) are mixed in a dry three-hole flask with a selective dissolution temperature of $100^{\circ} \mathrm{C}$ and a dissolution time of $4 \mathrm{H}$, The resulting solution is evenly spread in the glass tank. After vacuum exhaust, the table top is placed $1 \mathrm{H}$ and carefully poured into the distilled water to repeatedly soak the film. The cellulose regenerated film is placed on a clean glass sheet, dried at room temperature $2 \mathrm{H}$, covered with two pieces of glass, and then dried in a vacuum oven at $60{ }^{\circ} \mathrm{C}$ for $24 \mathrm{H}$. Finally, a yellowish translucent cellulose regenerated film is obtained, put in a desiccant to save for use [3].

\subsection{The DP of cellulose}

The degree of polymerization (DP) was determined using a standard procedure in cadoxen as described. $480 \mathrm{ml}$ distilled water and $220 \mathrm{ml}$ ethylenediamine were mixed evenly, and $54 \mathrm{~g}$ cadmium oxide was added under magnetic stirring in an ice bath. Continue to stir 1 hour, put the solution in the refrigerator for overnight preservation, then remove the solution and leave it at rest $1 \mathrm{H}$. Centrifugal remove the White precipitate to obtain a clear and transparent cadmium ethylamine solution. Accurately referred to as $0.0400 \mathrm{~g}$ of cellulose, add $20 \mathrm{ml}$ cadmium ethylenediamine solution, dissolve under magnetic stirring, and measure the polymerization of cellulose with a Ubbelodhe viscometer at $25^{\circ} \mathrm{C}$. The specific methods and precautions are described in the references. The formulas are as follows:

$$
\begin{aligned}
& \eta_{r}=\frac{\eta}{\eta_{0}} \approx \frac{t}{t_{0}} \\
& \eta_{s p}=\eta_{r}-1 \approx \frac{t}{t_{0}}-1 \\
& {[\eta]=\frac{\eta_{s p}}{C\left(1+0.29 \eta_{s p}\right)}} \\
& {[\eta]=3.85 \times 10^{-4} M \eta^{0.76}} \\
& D p=M \eta / 162
\end{aligned}
$$

\subsection{Study on the Degradation of Cellulose Regenerated Film}

The degradation of cellulose films with regeneration temperature of $100{ }^{\circ} \mathrm{C}$ and dissolution time of 3 hours was studied. The cellulose film is buried in the box in the soil with rot leaves near the root of the tree and kept moist. The degree of degradation was observed after 10 days and 15 days respectively.

\section{RESULTS AND DISCUSSION}

\subsection{Analysis of Cellulose Content of Corn Straw and Corn Core}

According to a study, the nitrate ethanol cellulose content of corn straw and corn cob were analyzed [4].
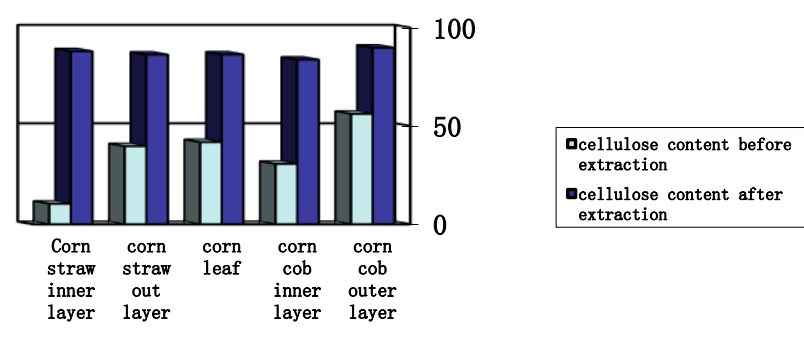

Figure 4: The nitrate ethanol cellulose content of corn straw and corn cob

Figure 4 shows that the content of ethanol nitrate cellulose in the inner layer of corn straw, the outer layer of corn straw, the leaf of corn, the inner layer of corn core, and the outer layer of corn core were $10.47 \%, 39.7 \%$, $41.75 \%, 30.7 \%$, and $56.16 \%$, respectively. The content of cellulose in the outer layer of corn straw is higher than the inner layer. The cellulose content in the outer layer of corn cob is higher than that in the inner layer of corn cob, so the cellulose content in the selection of corn straw and corn cob outer layer is higher and has a great value for utilization. In addition, the content of cellulose can be increased by the acid-base method. The cellulose content of these products is higher than $83 \%$ and can be used for the reproduction of cellulose.

\subsection{Dp Analysis of Corn Straw cellulose and Corn Core cellulose}

Dp analysis of cellulose content of corn straw and corn core, the results are shown in Figure 5.
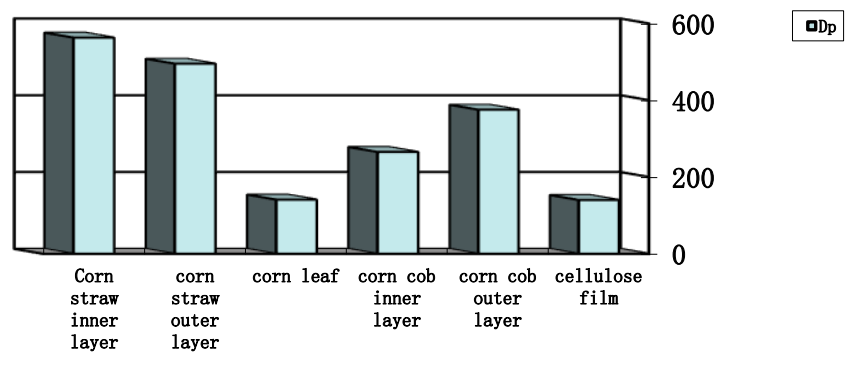

Figure 5: Cellulose content of corn straw and corn core

Figure 5 shows that the Dp of corn straw cellulose $>$ Dp of corn cob cellulose $>D p$ of corn leaf cellulose. The Dp of corn straw inner layer is greater than that of corn straw outer layer, and cellulose Dp of corn core outer layer > Dp of corn core inner layer. The Dp of cellulose film is lower than the Dp of cellulose.

\subsection{Study on the Degradation of Cellulose Regenerated Film}

A group of cellulose films is buried in the soil box with carrion leaves near the root of the tree and kept moist. The degree of degradation was observed after 10 days and 15 days respectively.

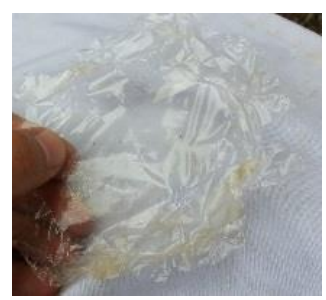

(a) before degradation

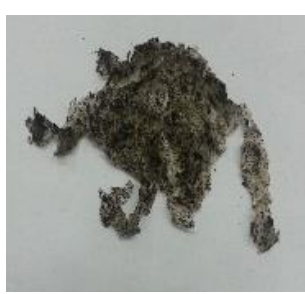

(a) 10 days later

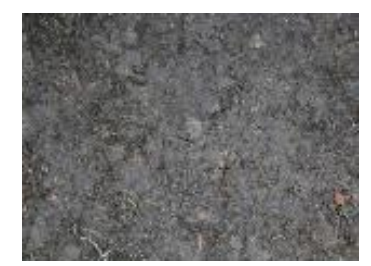

(c) 15 days later

Figure 6: Degradation of Cellulose Regenerated Film 
As can be seen from Figure 6, corn straw regenerated film can be degraded in the soil and completely degraded in just 15 days.

\section{CONCLUSIONS}

Using corn straw and corn cob as raw materials, cellulose was separated by acid and alkali method and dissolved in ionic liquid AmimCl forms a cellulose solution and regenerates in water to give a cellulose film. This study provides a chance to prepare Functional cellulose Regenerated film using relatively cheap and large amount of corn byproduct as cellulose resources.

\section{REFERENCES}

[1] Gu, Y.L., Shi, F., Deng, Y.Q. 2004. Research and application of ionic liquids in catalysis and separation. Journal of Chemical Industry and Engineering (China), 55 (12), 1957-1962.

[2] Wang, B., Cao, Y., Huang, K.L., 2010. Dissolution and regeneration of sugarcane bagasse cellulose in ionic liquid. Journal of Chemical Industry and Engineering (China). 61, (6), 1592-1598.

[3] Huang, K.L., Wang, B., Cao, Y. 2011. Homogeneous Preparation of Cellulose Acetate Propionate (CAP) and Cellulose Acetate Butyrate (CAB) from Sugarcane Bagasse Cellulose in Ionic Liquid. Journal of Agricultural and Food Chemistry, 59, 5376-5381

[4] Liu, S.C. 2004. Analysis and detection of pulping and papermaking. Beijing, Chemical industry press. 